\title{
Safety, immunogenicity and clinical efficacy of post exposure prophylaxis in confirmed rabies exposures
}

\author{
Ravish Haradanhalli S, Chandana Krishna, Pradeep Kumar DP, Iswarya Siddareddy and Rachana Annadani R \\ Department of Community Medicine, Kempegowda Institute of Medical Sciences (KIMS), India
}

\begin{abstract}
Many newer rabies immune biologicals are available in the market. Therefore, there is a need for periodic reinforcement of safety, immunogenicity and clinical efficacy of these in post exposure prophylaxis. All confirmed rabies exposures, during the period of 6 years from January 2010 to December 2015 , were retrieved. Assessment of safety of PEP was done by studying the documented immediate \& delayed; adverse drug reactions. Assessment of immunogenicity was done in 27 volunteered bite victims on Day 14 by modified RFFIT at the Department of Neurovirology, NIMHANS, Bangalore, India which is a WHO collaborating centre for reference and research on rabies.

The incidence of adverse drug events (ADEs) was found to be 7.5\%. All the ADEs were mild and resolved without any complications. All the 27 volunteered subjects had adequate RVNA concentration of $\geq 0.5 \mathrm{IU} / \mathrm{mL}$ on day 14 . The geometric mean RVNA concentration was $8.12 \mathrm{IU} / \mathrm{mL} \&$ all the $95 \mathrm{study}$ subjects were healthy and alive, after 1 year of completing post exposure prophylaxis. In conclusion, the presently available rabies immune biologicals were found to be safe, immunogenic and clinically efficacious for post exposure prophylaxis.
\end{abstract}

\section{Introduction}

Rabies is a fatal encephalitis that occurs in $>100$ countries throughout the world and poses a potential threat to $>3.3$ billion people worldwide [1]. It is a neglected zoonotic disease (a disease that is transmitted from animals to humans) caused by the rabies virus of the Lyssavirus genus, within the family Rhabdo viridae. The virus is transmitted by the saliva of rabid animals and generally enters the body via infiltration of virus-laden saliva from a rabid animal to other animals/humans through bites, scratches, licks on broken skin and mucous membrane [2]. Although a number of carnivores species serve as natural reservoirs, domestic dogs are the most common reservoir of the virus, with more than $95 \%$ of human deaths caused by dogmediated rabies [3].

A combination of large human and dog populations in congested habitable areas combined with widespread poverty has led to more exposures in World Health Organization (WHO)'s South East Asia Region than in any other part of the World. More than 1.4 billion people in this Region are at risk of rabies infection. Therefore, it continues to be a major public health and economic problem throughout the Region [4]. India is also a rabies endemic country, where animal bites to humans are a major public health problem and an estimated 17.4 million animal bites occur annually which accounts to an incidence of $1.7 \%[5]$.

Rabies is a $100 \%$ preventable disease. Timely and correct post exposure prophylaxis (PEP) for the exposed victims is necessary to prevent rabies. Every year, more than 15 million people worldwide receive PEP to prevent the disease and this is estimated to prevent hundreds of thousands of rabies deaths annually [1].

Post exposure prophylaxis should be started as early as possible after exposure, but in no case, should it be denied to exposed persons, after whatever the time interval has elapsed [4]. Proper wound management and simultaneous administration of rabies immunoglobulin (RIG) combined with anti-rabies vaccine (ARV) is almost invariably effective in preventing rabies, even after high-risk exposure.

Increased urbanization and ineffective dog population control measures have resulted in increased incidence of dog bites. Therefore, the demand for a safe and effective cell culture vaccines and rabies immunoglobulin for PEP is ever increasing. In this regard, many private pharmaceutical companies have started manufacturing rabies immune biologicals and these are available in the market. Therefore, there is a need for the periodic reinforcement of safety, immunogenicity and efficacy of post exposure prophylaxis in preventing rabies, more so, as newer anti-rabies vaccines and rabies immunoglobulins are becoming available in the market. Hence, this study was undertaken to throw more light on the existing information about the safety, immunogenicity and clinical efficacy of post exposure prophylaxis following confirmed rabies exposures.

\section{Methodology}

The study was conducted at anti rabies clinic, Kempegowda Institute of Medical Sciences (KIMS) Hospital and Research Centre, Bangalore, India. All the case records of confirmed rabies exposures, whose confirmation reports were available, during the period of 6 years from January 2010 to December 2015, were retrieved.

Correspondence to: Ravish Hardanahalli Shankaraiah, Associate Professor, Department of Community Medicine, Kempegowda Institute of Medical Sciences (KIMS), Banashankari 2nd Stage, Bangalore-560070, India, Tel: 9900562743, E-mail: drravishhs@rediffmail.com

Key words: rabies vaccines, rabies immunoglobulin, safety, immunogenicity, clinical efficacy

Received: June 11, 2016; Accepted: July 26, 2016; Published: July 29, 2016 
The confirmation of rabies in the biting animal was done at Government Veterinary Hospital, Bangalore by examination of brain samples for Negri-bodies using seller's stain/Fluorescent Antibody Test (FAT) and in those exposed to human rabies cases, the diagnosis of those cases were made on clinico-epidemiological basis at the epidemic disease hospital.

All 95 subjects, who had confirmed rabies exposures during the study period were included in the study and their case records were studied in detail regarding socio-demographic profile, type of exposure and post exposure prophylaxis provided.

\section{Assessment of safety}

It was done by studying by studying the documented immediate and delayed; local/ systemic adverse drug reactions (ADRs) on Days $0,3,7,14 \& 28$.

\section{Assessment of Immunogenicity}

27 of the bite victims had volunteered to get their rabies virus neutralizing antibody (RVNA) levels on Day 14 by modified rapid fluorescent focus inhibition test (RFFIT) at the Department of Neurovirology, National Institute of Mental Health and Neurosciences (NIMHANS), Bangalore, India which is a WHO collaborating centre for reference and research on rabies.

\section{Estimation of rabies virus neutralizing antibody (RVNA)}

Modified RFFIT was done as per WHO recommended procedure. The cell line used was BHK 21 (ATCC CCL 10) and 96 well tissue culture plates (Sigma) and BHK21 adapted CVS 11 strain of rabies virus. The reference serum used was an in house serum calibrated against 2nd international reference standard having a titer of $30 \mathrm{IU} / \mathrm{mL}$ (obtained from National Institute of Biological Standards).

Briefly, doubling dilutions of serum samples and reference serum (after heat inactivation at $56^{\circ} \mathrm{C}$ for $30 \mathrm{~min}$ in a water bath) in duplicate were made in 96 well plates using IMDM (Sigma Cat No.17633). To each $100 \mathrm{uL}$ of serum dilution $100 \mathrm{uL}$ of CVS $\left(100 \mathrm{FFD}_{50}\right)$ was added and the plate to was incubated at $37^{\circ} \mathrm{C}$ for $1 \mathrm{~h}$. A confluent monolayer of BHK 21 cells were trypsinized and re- suspended in $10 \mathrm{~mL}$ of IMDM with 10\% FCS (Sigma, cat No. F2442). Cell control and virus controls were also included. To each well of the 96 well plate $100 \mathrm{uL}$ of cell suspension was added and the plate was incubated at $37^{\circ} \mathrm{C}$ in a $\mathrm{CO}_{2}$ incubator (Sanyo). After $24 \mathrm{~h}$ the cells were fixed in cold acetone for $30 \mathrm{~min}$ and stained by direct FAT using commercially available rabies N conjugate (Light Diagnostics, Cat No. F199). The plates were then observed under an inverted fluorescence microscope (Nikon Eclipse). The highest dilution of serum showing $50 \%$ inhibition of fluorescence foci was taken as end point dilution. The titer was converted to IU/mL in comparison with reference serum.

All information pertaining to the study subjects were recorded which contained detailed information on socio demographic profile, relevant past and present medical history, anthropometry, physical and systemic examination findings, details of PEP provided, dates of vaccination, adverse drug reactions (ADR), treatment of ADRs, and RFFIT results.

Subsequently, all the study subjects were followed up through telephonic contact and wherever required by house visits to confirm their survival status, one year after receiving PEP following confirmed rabies exposure.
The local Epidemic Diseases Hospital, which admits human rabies cases, was also visited and its records were verified to rule out any possible rabies death amongst these subjects who received rabies PEP in KIMS Hospital.

\section{Statistical analysis}

The data was analyzed statistically by computing percentages, geometric mean concentration (GMC) and range.

\section{Results}

The present study included 95 subjects, who had confirmed rabies exposures. The mean age \pm standard deviation of the study subjects being $34.4 \pm 12.3$ years and most of them belonged to the age group of 14- 65 years. Majority of the study subjects were males (72.6\%) and from urban area (66.3\%) (Table 1). The biting animal was dog $(79.2 \%)$ in most of the cases followed by attendants of rabies patients and staff (8.4\%) of Epidemic Diseases Hospital (EDH) who were accidentally bitten/came in contact with the saliva of human rabies cases while attending/managing them in the hospital. Others included exposures to rabid cat, cow, horse and buffalo (12.4\%). Most of the exposures were category III $(91.6 \%)$ and on the limbs $(86.3 \%)$ with majority being either lacerations or abrasions $(74 \%)$. The immediate washing of wound(s) at home by water and soap and/or antiseptic by the rabies exposed persons was done only by $57.9 \%$ of the study subjects (Table 2 ).

\begin{tabular}{|l|c|}
\hline Characteristics & $\begin{array}{c}\text { Study Subjects } \\
(\mathbf{n = 9 5 )}\end{array}$ \\
\hline Age (mean \pm S.D) years & $\mathbf{3 4 . 4} \pm \mathbf{1 2 . 3}$ \\
< 14 years & $25(26.3 \%)$ \\
14- 65 years & $67(70.5 \%)$ \\
$>$ 65 years & $03(03.2 \%)$ \\
\hline Sex: & \\
Male & $69(72.6 \%)$ \\
Female & $26(27.4 \%)$ \\
\hline Place of residence: & \\
Urban & $63(66.3 \%)$ \\
Rural & $32(33.7 \%)$ \\
\hline
\end{tabular}

Table 1. Socio demographic characteristics of the study population.

Table 2. Details of exposure in the study population.

\begin{tabular}{|l|r|}
\hline Details of exposure & $\begin{array}{c}\text { Study Subjects } \\
\text { (n=95) }\end{array}$ \\
\hline Exposure: & $75(79.2 \%)$ \\
Dog & $08(08.4 \%)$ \\
Human case & $03(03.1 \%)$ \\
Cat & $03(03.1 \%)$ \\
Horse & $03(03.1 \%)$ \\
Cow & $03(03.1 \%)$ \\
Buffalo & \\
\hline Site of bite: & $42(44.2 \%)$ \\
Lower limb & $40(42.1 \%)$ \\
Upper limb & $9(09.5 \%)$ \\
Trunk & $4(04.2 \%)$ \\
Head \& Neck & \\
\hline Type of wound: & $45(47.4 \%)$ \\
Lacerations & $25(26.3 \%)$ \\
Abrasions & $17(17.9 \%)$ \\
Punctured wound & $8(08.4 \%)$ \\
\hline Licks & \\
\hline Categorization of wound & $8(08.4 \%)$ \\
Cat II & $87(91.6 \%)$ \\
\hline Cat III & \\
\hline Immediate wound wash: & $55(57.9 \%)$ \\
Yes & $40(42.1 \%)$ \\
\hline No & \\
\hline
\end{tabular}


In the hospital, all the bite victims had received complete PEP as per the recommendations of WHO, which included thorough wound wash and all category III exposures were given total quantity of required rabies immunoglobulin i.e., HRIG (6.4\%) or ERIG (93.6\%) locally, into and around all the wound/s.

Similarly, all the study subjects were given full course of anti-rabies vaccination, intramuscularly by Essen regimen using various types of vaccines viz. PCECV (81.1\%), PVRV (12.5\%), PDEV (3.2\%) and HDCV (3.2\%) (Table 3).

The common ADEs were erythema at the site of injection, itching at the site of injection, pain at the site of injection and induration at the site of injection as shown in table 4 . The incidence of adverse drug events (ADEs) was calculated based on number of ADEs reported and recorded, divided by total number of vaccine doses given and it was found to be $7.5 \%(36 / 475)$. All the ADEs were local in nature and there were no systemic reactions. All the ADEs were mild and resolved without any complications.

The RVNA response was tested among 27 volunteered subjects on day 14 and all the study subjects had adequate RVNA concentration of $\geq 0.5 \mathrm{IU} / \mathrm{mL}$. The geometric mean RVNA concentration (GMC) was $8.12 \mathrm{IU} / \mathrm{mL}$.

All the 95 study subjects were healthy and alive, after 1 year of completing post exposure prophylaxis.

\section{Discussion}

Rabies is a neglected zoonotic disease that is insufficiently addressed by Governments and the International community, as they are best defined by the people and communities they affect the most i.e., poor people living in remote rural areas and urban slums of the developing World. It is however, the disease most amenable to control, as the tools for prevention i.e., post exposure prophylaxis are available. Therefore, it is the first zoonosis on the list of neglected diseases targeted for regional and eventually global elimination.

India is highly endemic for rabies and has the largest number of animal bites in the World. Therefore, in rabies endemic country like

Table 3. Details of post exposure prophylaxis provided at the hospital.

\begin{tabular}{|l|c|}
\hline Post exposure prophylaxis & $\begin{array}{c}\text { Study Subjects } \\
(\mathbf{n}=\mathbf{9 5})\end{array}$ \\
\hline Wound wash: Done & $95(100 \%)$ \\
\hline Anti-rabies vaccine used: & $77(81.1 \%)$ \\
PCEC & $12(12.5 \%)$ \\
PVRV & $3(03.2 \%)$ \\
PDEV & $3(03.2 \%)$ \\
\hline HDCV & Cat III (87) \\
\hline Rabies immunoglobulin used & $6(06.3 \%)$ \\
HRIG & $81(93.7 \%)$ \\
ERIG & $75(86.2 \%)$ \\
Site of infiltration: & $12(13.8 \%)$ \\
Local & \\
Both local \& systemic & \\
\hline
\end{tabular}

Table 4. Adverse drug events among the study subjects.

\begin{tabular}{|l|c|}
\hline Adverse drug events (Local) & Study subjects $(\mathbf{n = 9 5})$ \\
\hline Pain & $12(2.5 \%)$ \\
\hline Itching & $11(2.3 \%)$ \\
\hline Erythema & $10(2.1 \%)$ \\
\hline Induration & $3(0.6 \%)$ \\
\hline Total & $\mathbf{3 6} / \mathbf{4 7 5 *}(\mathbf{7 . 5 \% )}$ \\
\hline
\end{tabular}

*95 subjects X 5 doses
India, where every animal bite is potentially suspected as a rabid animal bite, the treatment should be started immediately. Because of long incubation period, which is typical of most cases of human rabies, it is possible to institute post exposure prophylaxis to ensure that the individual will be immunized before the rabies virus reaches the nervous system.

In India, increased urbanization and ineffective dog population control measures have resulted in increased incidence of dog bites. Therefore, the demand for a safe and effective cell culture vaccines and rabies immunoglobulin for PEP is ever increasing. In this context, many pharmaceutical companies have started production and the availability of these newer vaccines and immunoglobulins for PEP are always present in the market [6-11]. Therefore, the safety and efficacy of these rabies immune biologicals should be established periodically.

All CCEEVs induce a prompt and high rabies-virus neutralizing antibody response to the viral G protein, which will be above WHO's specified minimum titre of $0.5 \mathrm{IU} / \mathrm{ml}$ of serum, measured by RFFIT. In healthy vaccinees, this level should be achieved in most individuals by day 14 of a post-exposure regimen, with or without simultaneous administration of rabies immunoglobulin and irrespective of age.

The present study assessed the safety of all the study subjects and 27 of them volunteered to get their RVNA estimation done. Ideally the efficacy of rabies PEP is assessed by rabies virus neutralizing antibodies (RVNA) assay in all the bite victims. But, RVNA assays are expensive, scarce, practically not feasible and also not recommended as a matter of routine in all cases. In these circumstances, it is the survival of the exposed persons after rabies PEP and beyond the usual incubation period of the disease, is an indicator of the clinical efficacy of treatment. This is generally considered as six months, as is evident by the WHOAPCRI national multi-centric rabies survey. The present study showed that all the 95 rabies exposed persons were successfully treated at the ARC, KIMS Hospital, Bangalore and were alive and healthy even after 1 year. Similarly, a study done in Philippines among patients severely exposed to laboratory-confirmed rabid animals showed that, the postexposure rabies prophylaxis was safe and efficacious [12].

In conclusion, the present study showed that, the presently available rabies immune biologicals i.e., anti-rabies vaccines and rabies immunoglobulin were found to be safe, immunogenic and clinically efficacious for post exposure prophylaxis.

\section{References}

1. WHO Expert Consultation on Rabies (2013) Second report. Technical Report Series No. 982. Geneva: World Health Organization.

2. World Health Organization (2010) Rabies vaccines: WHO position paper, Weekly Epidemiological Record, No. 32 85: 309-20.

3. WHO Expert Consultation on Rabies (2005) First report. Technical Report Series No. 931 Geneva: World Health Organization.

4. WHO South East Asia region: Strategic Framework for Elimination of Human Rabies Transmitted by Dogs in the South-East Asia Region: World Health Organization, Regional office for South East Asia; 2012

5. Sudarshan MK, Madhusudana SN, Mahendra BJ, Rao NS, Ashwath Narayana DH, et al. (2007) Assessing the burden of human rabies in India: results of a national multicenter epidemiological survey. Int J Infect Dis 11: 29-35. [Crossref]

6. Sehgal S, Bhattacharya D, Bhardwaj M (1995) Ten year longitudinal study of efficacy and safety of purified chick embryo cell vaccine for pre exposure and post exposure prophylaxis of rabies in Indian population. J Commun Dis 27: 3-43. [Crossref]

7. Ashwath Narayana DH, Madhusudana SN, Sampath G, Sudarshan MK, Gangaboraiah, et al. (2014) Safety and immunogenicity study of a new purified chick embryo cell rabies vaccine Vaxirab-N (Pitman-Moore strain) manufactured in India. Hum Vaccin Immunother 10: 120-125. [Crossref] 
8. Ashwathnarayana DH, Madhusudana SN, Sampath G, Sathpathy DM, Mankeshwar $R$, et al. (2009) A comparative study on the safety and immunogenicity of Purified duck embryo vaccine (PDEV, Vaxirab) with purified chick embryo cell vaccine (PCEC, Rabipur) and purifed vero cell rabies vaccine (PVRV, Verorab). Vaccine 28: 148-151. [Crossref]

9. Sampath G, Madhusudana SN, Sudarshan MK, Ashwathnarayana DH, Mahendra BJ, et al. (2010) Immunogenicity and safety study of Indirab: a Vero cell based chromatographically purified human rabies vaccine. Vaccine 28: 4086-4090. [Crossref]

10. Sampath G, Reddy SV, Rao ML, Rao YU, Palaniappan C (2005) An immunogenicity study of a newly introduced purified Vero cell rabies vaccine (Abhayrab) manufactured in India. Vaccine 23: 897-900. [Crossref]
11. Sudarshan MK, Bhardwaj S, Mahendra BJ, Sharma H, Sanjay TV, et al. (2008) An immunogenicity, safety and post-marketing surveillance of a novel adsorbed human diploid cell rabies vaccine $\left(\operatorname{Rabivax}^{\circledR}\right)$ in Indian subjects. Human Vaccines 4: 275-279. [Crossref]

12. Quiambao BP, Dimaano EM, Ambas C, Davis R, Banzhoff A, et al. (2005) Reducing the cost of post-exposure rabies prophylaxis: efficacy of $0.1 \mathrm{ml}$ PCEC rabies vaccine administered intradermally using the Thai Red Cross post-exposure regimen in patients severely exposed to laboratory-confirmed rabid animals. Vaccine 23: 1709-1714. [Crossref]

Copyright: (C2016 Ravish Haradanhalli S. This is an open-access article distributed under the terms of the Creative Commons Attribution License, which permits unrestricted use, distribution, and reproduction in any medium, provided the original author and source are credited. 